\title{
GRANULARITY OF EFFICIENT ENERGY SAVING IN WIRELESS SENSOR NETWORKS
}

\author{
M. Chithik Raja \\ Salalah College of Technology - Oman, chithik25@gmail.com
}

\begin{abstract}
Most existing techniques for reconfigurable processors focus on the computation model. This paper focuses on increasing the granularity of Efficient Energy Saving units without compromising flexibility. This is carried out by matching the granularity to the degree-of-freedom processing in most wireless Networks. A design flow that accelerates the exploration of tradeoffs among various architectures for the configurable unit is discussed in this paper. In this Paper we mentioned in detailed way of evaluates various baseband algorithms and presents the granularity of configurable units, describes the architecture of each configurable unit and presents the methodology used in the architectural tradeoff analyses as well as the tradeoff results.
\end{abstract}

Keywords: Wireless Communication, Low Power Design, OFDM, MIMO.

\section{INTRODUCTION}

A wireless network is a flexible data communications system, which uses wireless media such as radio frequency technology to transmit and receive data over the air, minimizing the need for wired connections. Wireless networks are used to augment rather than replace wired networks and are most commonly used to provide last few stages of connectivity between a mobile user and a wired network. Wireless networks use electromagnetic waves to communicate information from one point to another without relying on any physical connection. Radio waves are often referred to as radio carriers because they simply perform the function of delivering energy to a remote receiver. The data being transmitted is superimposed on the radio carrier so that it can be accurately extracted at the receiving end. Once data is superimposed (modulated) onto the radio carrier, the radio signal occupies more than a single frequency, since the frequency or bit rate of the modulating information adds to the carrier. Multiple radio carriers can exist in the same space at the same time without interfering with each other if the radio waves are transmitted on different radio frequencies. To extract data, a radio receiver tunes in one radio frequency while rejecting all other frequencies. The modulated signal thus received is then demodulated and the data is extracted from the signal. Wireless networks offer the following productivity, convenience, and cost advantages over traditional wired networks:

- Mobility: provide mobile users with access to real-time information so that they can roam around in the network without getting disconnected from the network. This mobility supports productivity and service opportunities not possible with wired networks.
- Installation speed and simplicity: installing a wireless system can be fast and easy and can eliminate the need to pull cable through walls and ceilings.

- Reach of the network: the network can be extended to places which cannot be wired

- More Flexibility: wireless networks offer more flexibility and adapt easily to changes in the configuration of the network.

- Reduced cost of ownership: while the initial investment required for wireless network hardware can be higher than the cost of wired network hardware, overall installation expenses and life-cycle costs can be significantly lower in dynamic environments.

- Scalability: wireless systems can be configured in a variety of topologies to meet the needs of specific applications and installations. Configurations can be easily changed and range from peer-to-peer networks suitable for a small number of users to large infrastructure networks that enable roaming over a broad area.

Bluetooth and $802.11 \mathrm{~b}$ have the potential to dramatically alter how people use devices to connect and communicate in everyday life. Bluetooth is a low-power, short-range technology for ad hoc cable replacement; it enables people to wirelessly combine devices wherever they bring them. Conversely, $802.11 \mathrm{~b}$ is a moderate-range, moderate-speed technology based on Ethernet; it allows people to wirelessly access an organizational network throughout a campus location. Although the technologies share the $2.4 \mathrm{GHz}$ band, have some potentially overlapping applications, and have been pitted against each other in the press, they do not compete and can even been successfully combined for corporate use. 
The reconfiguration mechanism is similar to Rapid. Control signals are divided into hard control and soft control. The hard control signals are similar to those in an field-programmable gate array (FPGA) and change infrequently. The soft control signals are similar to those in microprocessors and change almost every clock cycle. The hard control signals have fixedlength instructions, while the soft control signals have variable-length instructions as only a small portion of them is used in any computation. Finally, a prototype of the processor is implemented using the Intel 0.13- m CMOS standard cell library at $1.2 \mathrm{~V}$. It consists of an array of nine configurable units: four dominant DOF units, two interconnect units, and three accelerators for coordinate transformation, maximumlikelihood (ML) detection, and miscellaneous arithmetic operations. The interconnect units are specifically designed to support pipeline and stream processing so as to further enhance the energy efficiency. They utilize the timemultiplexed cross-bar architecture to substantially reduce the number of wires. Therefore, the entire baseband processor is a multi rate system. The interconnect and the memory run at $200 \mathrm{MHz}$, while most of the configurable units run at 50 MHz's The total gate count is $569 \mathrm{e} 3$ and the estimated power consumption is $63.4 \mathrm{mw}$. The energy efficiency is 95 $\mathrm{MOP} / \mathrm{mW}$, which is in the same order as dedicated hardware implementations. orders of magnitude more power efficient than digital signal processors (DSP). Most existing reconfigurable architectures are in between these two extremes, and are usually more towards the DSPs.

\section{SELECTION OF GRANAULITY}

Fig. 1 shows the DOF processing (also known as symbol processing) of three popular receiving systems: DS-CDMA, OFDM, and multiple-antenna OFDM. DS-CDMA is part of the $3 \mathrm{G}$ cellular standard and the IEEE $802.11 \mathrm{~b}$ wireless LAN standard. OFDM is part of the IEEE 802.11a/g wireless LAN standard and the IEEE802.16e wireless MAN standard. Multiple-antenna OFDM is part of the IEEE 802.11 in highthroughput wireless LAN standard. In DS-CDMA systems the incoming signal first goes through a matched filter and then correlates with either its delayed replica or a training sequence during synchronization. Once a signal is detected, the estimated timing offset will be compensated. Afterward, the signal is despread with the PN sequence to obtain modulated data symbols. Similar to DS-CDMA systems, the incoming signal of OFDM systems first goes through a decimation filter followed by either autocorrelation or cross correlation for synchronization. Upon detection of a signal, the estimated frequency offset is corrected and then data symbols are demodulated by the FFT operation. Finally, the multipleantenna OFDM system is composed of a parallel of several OFDM systems. The demodulated symbols after the FFT operation are combined according to the space-time coding schemes used, such as the V-BLAST and the SVD-based algorithms.
TABLE I GRANULARITY OF CONFIGURABLE UNITS AND
OPERATIONS SUPPORTED

\begin{tabular}{|l|l|}
\hline Configurable Units & Operation Supported \\
\hline DOF (Domain) & $\begin{array}{l}\text { Dispreading } \\
\text { Matrix Vector Multiply } \\
\text { FIR filter } \\
\text { Cross-Correlation } \\
\text { Euclidean distance } \\
\text { Calculation } \\
\text { N-point FFT } \\
\text { Auto Correlation }\end{array}$ \\
\hline CORDIC & $\begin{array}{l}\text { Normalization } \\
\text { sine, cosine, Asine } \\
\text { Acosine }\end{array}$ \\
\hline ML & \begin{tabular}{l} 
Maximum likelihood \\
\hline ALU
\end{tabular} \\
\hline
\end{tabular}

The bottom of Fig. 1 summarizes the operations involved. To support all the operations by an array of homogeneous configurable units would make the configurable unit too bulky. Instead, we group the most frequent and similar operations together to be supported by an array of dominant units.Each unit is called the DOF configurable unit. All remaining operations will be supported by three other configurable units: a coordinate rotation digital computer (CORDIC), an ML accelerator, and a dual-core ALU. Table I summarizes the classification of operations. As a whole, Fig. 2 shows the architecture of the reconfigurable baseband processor. The receiver chains shown in Fig. 1 also reveal the stream based processing of most baseband algorithms in wireless communication. The better we preserve this property in the implementation, the more power efficient the processor. The power efficiency is derived from maintaining data locality. 


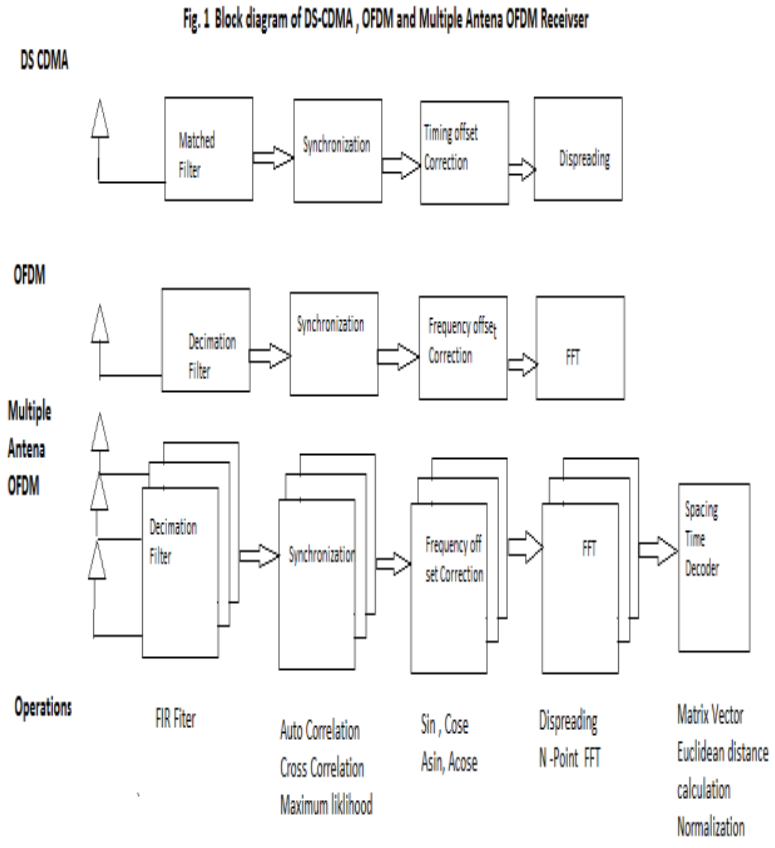

In our interconnect configurable units, the outputs from the array of data path configurable units can be configured either to feed back to the memory or to be inputs to the array. This is illustrated in the right side of Fig. 2, where interconnect to the data path has inputs from memory or from outputs of the data path. To give an idea, the interconnect units are designed such that they can be configured into a pipelined datapath illustrated in Fig. 2.

\section{Fig. 2 Macro architecture of the reconfigurable processor}

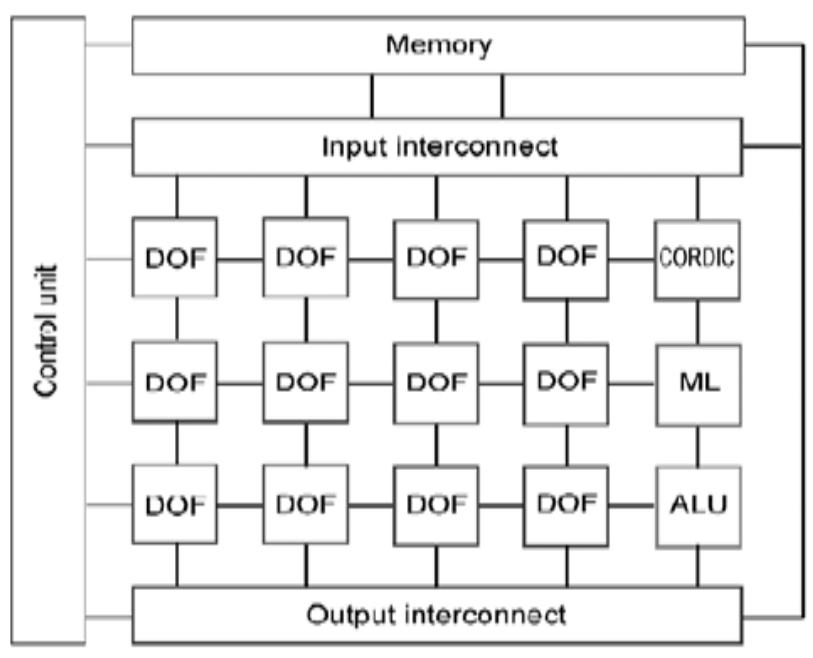

Fundamental problem in any communication system is: given a communication channel between a transmitter and a receiver, how many "independent" signals can be exchanged between them? Arbitrary communication channels that can be described by linear compact channel operators mapping between normed spaces are examined in this paper. The (wellknown) notions of degrees of freedom at level and essential dimension of such channels are developed in this general setting. We argue that the degrees of freedom at level \$lepsilon\$ and the essential dimension fundamentally limit the number of independent signals that can be exchanged between the transmitter and the receiver. We also generalize the concept of singular values of compact operators to be applicable to compact operators defined on arbitrary normed spaces which do not necessarily carry a Hilbert space structure. We show how these generalized singular values can be used to calculate the degrees of freedom at level \$lepsilon\$ and the essential dimension of compact operators that describe communication channels. We describe physically realistic channels that require such general channel models.

\section{RELAY AND COOPERATIVE}

\section{COMMUNICATIONS}

Relay and cooperative networks are also promising architectures to improve EE. Relay networks save energy in two ways: reducing path loss due to the shorter transmission range and potentially generating less interference due to the low transmission power. The transmission with relays can reduce energy consumption in CDMA cellular networks; and the higher the path loss exponent, the more energy that can be saved. Power control can further reduce energy consumption. The advantages of relay transmission are examined in where the transmission delay and energy consumption of relay nodes are both considered. The tradeoff between total energy consumption and end-to-end communication rate in AWGN relay channels is analyzed, where the impact of the hop number, node locations, allocated power and data rate of each hop on EE is also studied. Different from pure relay systems, each cooperative node in cooperative communications acts as both an information source and a relay. Inherently, energy savings in cooperative networks come from the diversity that results from cooperation. Since a relay node usually has a several-wavelength long distance to the source node, the relay channel experiences fading conditions independent of that on the direct channel between the source and destination. Hence, we can exploit the channel diversity for potential energy savings. A short distance, direct communication is preferred over distributed space-time coding since adding more energy consumption from using relays counters the transmission energy saving from cooperative diversity. However, the results in also demonstrate that the energy efficiency of cooperative communication can be improved by optimizing the constellation size for different transmission distances and outperform direct communication. Similarly, the research results in also show the optimal constellations in terms of 
Deere decreasing with transmission distances, where the energy efficiency of cooperative transmission using different MQAM schemes is investigated by jointly considering transmit, circuit, and retransmission power. It is found in that the single-best relay selection scheme with physical layer power allocation and the RTS/CTS 4 based MAC-layer can achieve higher EE compared with multiple relays. In the paper, two power control strategies are also introduced: minimizing energy consumption per data packet, or maximizing the network lifetime. It is shown that with the two power control schemes, he single best relay selection scheme can outperform direct communication and prolong the network lifetime. An energy efficient clustered wireless sensor network is studied in this paper, multiple relay nodes which correctly decode the broadcast message from the source, participate in relaying data packets using space-time coding. The optimal power allocation for intra-cluster and inter-cluster transmissions is derived and the effect of the number of relay nodes in the cluster, the average PER (Packet Error Rate) constraint, and the distance between clusters is investigated. The proposed schemes significantly improve the energy savings compared with direct communication. Another very popular technique- network coding- has also been used to implement cooperative communication. Network coding based two-way relay schemes with decoding (decode and-forward) and without decoding (amplify-and-forward, DE noise-andforward, compress-and-forward) are introduced and described. In these approaches, relay nodes first encode the received packets from different traffic flows into one packet and then forward the encoded packets to neighbors, instead of forwarding each packet individually. This leads to fewer transmissions, and thus potentially reduces energy consumption. The research work in shows that the potential gain of network coding in terms of EE is upper bounded by a factor of 3 a network coding-based cooperative diversity scheme in OFDMA wireless networks called 'XOR-CD' is proposed; the results show that 'XOR-CD' can improve the energy efficiency by over $100 \%$ compared with conventional cooperative schemes. However, the gain from network coding is closely related to that from exploiting traffic patterns, scheduling strategies, network topology, and so on. Hence, energy-efficient network design through network-codingbased cooperation is worth

\section{MIMO, OFDMA AND CROSS-LAYER DESIGN FOR ENERGY EFFICIENCY}

Both MIMO and OFDM are key techniques in current 3G and future $4 \mathrm{G}$ wireless systems, such as Worldwide Interoperability for Microwave Access (WiMAX) and the Third Generation Partnership Project (3GPP) Long Term Evolution (LTE). Previous research on MIMO and OFDM mainly aims to increase network capacity or spectral efficiency, but rarely concerns energy consumption. Thus, how to design energy efficient schemes with MIMO and OFDM is highly interesting and important. In this section, we will provide an overview on the state of the art on this issue, along with cross-layer optimization strategies for energyefficient wireless networks.

\section{A. MIMO}

MIMO can provide diversity gain and multiplexing gain. Particularly, diversity gain is achieved by sending signals that carry the same information through different paths between transmit antennas and receiver antennas. Multiplexing gain can be obtained by transmitting independent information streams in parallel through the spatial channels. Both help increase network throughput and reduce energy consumption. The impact of diversity gain and multiplexing gain on $\mathrm{EE}$ of MIMO transmission in wireless sensor networks is investigated. With MIMO transmission, more antenna devices will consume more circuit power. Therefore, MIMO is not always more energy-efficient than single-input and single output(SISO). An energy consumption model of MIMO in considers all signal processing blocks at the transmitter and the receiver. Based on this model, the relationship between energy consumption and transmission distance with SISO, SIMO and MIMO is investigated. It is shown that, at a short instance, SISO may outperform MIMO in EE because circuit energy consumption dominates the total energy demand. The trade-off between circuit power and transmission power consumption to obtain higher EE in MIMO systems is also discussed where the optimal MIMO mode with a required transmission rate is given. In a similar context, the adaptive MIMO switching strategy based on the available CSI, proposed can achieve up to a $30 \%$ improvement in link energy efficiency. In cooperative MIMO and data aggregation techniques are combined to reduce the energy consumption in wireless sensor networks; this is accomplished by reducing the amount of transmitted data and by better allocating resources through cooperation. The problem of energy-efficient MIMO preceding is considered for a point-topoint communication system with multiple antenna terminals .In power allocation in wireless ad hoc networks is structured as a non-cooperative game to maximize EE; and, a link shut-down mechanism is proposed to reduce co-channel interference and improve EE. In many practical systems, user terminals are usually equipped with only one antenna. Thus, traditional MIMO cannot be implemented. To overcome the limitation, multiuser MIMO (MU-MIMO), also called virtual MIMO, has been proposed. In MU-MIMO, multiple users cooperate for distributed transmission and information processing. Thus, local information exchange is indispensable for MU-MIMO. This causes additional energy consumption compared with traditional single-user MIMO (SU-MIMO). This issue has been studied. It is proved that, even when the local energy consumption for cooperation is considered, MU-MIMO is still more energy-efficient than SISO over a certain transmission distance. It is also shown that optimizing the constellation size can further increase EE. For both SU-MIMO and MU-MIMO, knowledge of the CSI is required. However, most of the mentioned work ignores the energy consumption of CSI 
signaling information. Thus, when the energy consumption of signaling information is considered, there may exist trade-off between the CSI accuracy and total energy efficiency.

\section{B.OFDMA}

Orthogonal frequency division multiple access (OFDMA) will be the dominant multiple access scheme for next generation wireless networks since both of the two accepted $4 \mathrm{G}$ This article has been accepted for inclusion in a future issue of this journal. Content is final as presented, with exception of pagination. standards (Long Term Evolution-Advanced and $802.16 \mathrm{~m}$ ) have adopted OFDMA as the multiple access technology. OFDMA is distinguished by its simplicity and high spectral efficiency. In this approach, multiple access is achieved by allocating different sets of orthogonal subcarriers to different users. The benefit is that subcarriers can be adaptively allocated to the users that experience high SNR. Hence, system capacity can be greatly increased. This is also known as multi-user diversity. Obviously, in OFDMA systems, multi-user diversity can be exploited not only to increase network capacity but also to reduce energy consumption. When a "good" channel is allocated to the corresponding user, the transmit power can be drastically decreased. Based on the above observation, an optimal subcarrier, bit, and power allocation algorithm minimizing the total transmit power. The optimal resource allocation scheme is to reduce the transmit power by about $5-10 \mathrm{~dB}$ compared with conventional schemes if circuit energy consumption is not considered. The impact of transmission rate, transmit energy and circuit energy consumption as well as channel gain on the EE in OFDMA systems is analyzed, where flat fading channels are considered. It is proved that, for a given channel gain and constant circuit energy consumption, there exists a unique globally optimal transmission rate in terms of EE. It is also proved that $\mathrm{EE}$ increases with the channel gain and the number of sub channels, while decreasing with the circuit energy consumption. Based on these observations, the authors further propose energy-efficient link adaptation (rate and corresponding transmit power) and resource allocation (subcarriers) scheme for OFDMA systems. Simulation results how that the EE scheduler performs approximately $50 \%$ better than a round-robin scheduler in terms of EE. The work is later extended to the case of frequency-selective channels. An energy-efficient water-filling power allocation scheme is proposed, where it is proved that the maximal EE can be achieved by adapting both overall transmit power and its allocation according to the channel states and the circuit energy consumption. A closed-form solution of energyefficient link adaptation is obtained with a time-averaged bitsper-Joule metric, where it is shown that this scheme can achieve almost the same performance as the global optimum which is obtained by exhaustive search, and with much ess complexity. An energy-efficient link adaptation strategy for MIMO-OFDM based wireless communications where the optimal mode is chosen to maximize EE with QoS constraint. In OFDMA systems, fairness among users is an important design standard. Adaptive resource allocation without considering fairness may cause poor service to some users since resource is always distributed to users with relatively high channel gains. Hence, when adaptive strategies are used to maximize energy efficiency, fairness should be taken into account. The fairness issue is considered where a geometricmean metric is proposed to guarantee that the sub channels are allocated to maximize the geometric average of the energy efficiency of all the users. Note that in the above discussion, the energy consumption of the signaling overhead is not considered. On the other hand, for an adaptive scheme, accurate CSI is imperative. Thus, the effect of signaling overhead on energy-efficient OFDMA design should be further studied. Additionally, when relay strategies are used in an OFDMA system, energy-efficient source allocation may become more intricate; this will also require further investigation.

\section{Cross-layer Optimization}

As indicated in cross-layer design is another prominent approach to reduce energy consumption. The design requirements for energy efficiency across the link, medium access, network and application layers have been investigated. A comprehensive discussion of energy-efficient cross layer design in the time, frequency, and spatial domains, as well as details related to energy-efficient hardware implementation provided. It is shown that, because each layer of the protocol stack has an inherent interdependence another layer, crosslayer strategies can significantly improve EE through adaptive transmission and resource allocation schemes corresponding to service, traffic, and environment dynamics. From the previously discussed strategies, it is also easy to see that crosslayer design plays a key role in reducing the holistic energy consumption, especially for networks with MIMO and OFDMA transmission schemes. Cross-layer design from a system-wide perspective brings low design margins. At the same time, it also leads to higher algorithm complexity. As a result, significant computational overhead is expected to obtain the optimal solution. The margin adaptive optimization problem, which aims at minimizing the overall transmitting power of users with individual rate constraints in realistic OFDMA systems, is NP-hard. Moreover, three suboptimal approaches (relaxation constraint, problem splitting, and heuristics) have been proposed to obtain nearly optimal solutions. Obviously, the cross-layer optimization also should consider signaling overhead.

\section{CONCLUSIONS}

In this article, we outlined the technical granularity efficient energy saving in wireless sensornetworks, and discussed the state-of-the-art research on energy efficient wireless networks. EE metric, network deployment strategies, energy-efficient network resource management, various relay and cooperative communications, MIMO and OFDM technologies, as well as cross-layer optimizations for developing energy-efficient 
wireless networks were introduced. For readers to easily find interesting references which have been presented in this paper, we have split the techniques discussed into those relevant to the base station site, those relevant to the terminal site, and those relevant to the network. Research shows that optimized energy-efficient design (including network deployment, transmission scheme and resource management) could significantly reduce the energy consumption of the entire network.

\section{REFERENCE}

[1] S. ZHU, S. SETIA, AND S. JAJODIA, LEAP: efficient security mechanisms for large-scaledistributed sensor networks, in 10th ACM conference on Computer and communication security (CCS'03), ACM Press, 2003, pp. 62-72.

[2] R. J. WATRO, D. KONG, S. FEN CUTI, C. GARDINER, C. LYNN, AND P. KRUUS,Tinypk: securing sensor networks with public key technology., in 2nd ACM Workshop on Security of ad hoc and Sensor Networks (SASN'04), Washington, DC, October 2004, pp.59-64.

[3] C. SHU, S. KWON, AND K. GAJ, FPGA accelerated Tate pairing based cryptosystemsover binary fields, in Cryptology Eprint Archive, Report 2006/179, 2006.

[4] Hayashibara, N., Cherif, A., and Katayama, T. Failure detectors for large-scale distributed systems. In Proc. of the 21st Symposium on Reliable Distributed Systems (SRDS'02) (2002), p. 404.

[5] Hua, C., and Zheng, R. Starvation modeling and identification in dense 802.11 wireless community networks. In Proc. of the 27th Joint Conference of the IEEE Computer and Communications Societies (INFOCOM'08) (2008), pp. 1022-1030.

[6] Information Processing Technology Office (IPTO) Defense Advanced Research Projects Agency (DARPA). Baa 07-46 landroids broad agency announcement. http://www.darpa.mil/IPTO/solicit/open/BAA-07-46 PIP.pdf, 2007.

[7] Manshaei, M. H., Freudiger, J., Felegyhazi, M., Marbach, P., and Jean- PierreHubaux. On wireless social community networks. In Proc. of the 27th Joint Conference of the IEEE Computer and Communications Societies (INFOCOM'08) (2008), pp. 15521560.

\section{BIOGRAPHIES:}

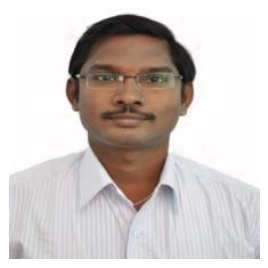

I am (Mr. Chithik Raja M.) studied Master of Engineering in Computer Science and Engineering at Respected Anna University Chennai Affiliated College R.V.S.C.E.T. Dindigul , Tamil Nadu , India. I am currently working on Salalah College of Technology, Oman. I am font of writing IT Books and Research article in reputed International IT Journals. I have published more than 10
Research Articles in different International Journals and published three IT Books with German Publisher Lambert Academic Publisher. I am very much interest to conducting work shop in Modern IT challenges and Software architectural Design. 